\title{
A TEST OF EARTHQUAKE EARLY WARNING SYSTEM USING LOW COST ACCELEROMETER IN HUALIEN, TAIWAN
}

\author{
Y.-M. Wu ${ }^{1}$, T.-L. Lin $^{2}$ \\ ${ }^{1}$ Department of Geosciences, National Taiwan University, 10617 Taipei, Taiwan, E-mail: \\ drymwu@ntu.edu.tw \\ ${ }^{2}$ Department of Earth Sciences, National Cheng Kung University, 70101 Tainan, Taiwan
}

Abstract

The earthquake early warning (EEW) research group at the National Taiwan University (NTU) and one technology company have been developing a Micro Electro Mechanical Systems (MEMS) type of accelerometer (the "Palert" EEW sensor) specifically designed for EEW purpose. In addition to the physical properties of the MEMS accelerometer, the main advantage of the MEMS accelerometer comparing to the other seismometers is that it is a relatively very low-cost seismic sensor. We present the performances of the Palert EEW network located at the Hualien region during a two-month experiment. The results of the Hualien Palert network encourage the further implementations for the MEMS-type of seismometer in the EEW application.

\section{Introduction}

Taiwan has been constantly threatened by large, devastating earthquakes as the consequence of the persistent collision between the Philippine Sea Plate and Eurasian Plate. For Taiwan, the development of earthquake early warning system was first motivated by the Hualien offshore earthquake $\left(M_{\mathrm{w}}=7.8\right)$ in November 15 , 1986, which caused severe damage in the metropolitan Taipei of about $120 \mathrm{~km}$ away from the epicenter. If an EEW system in the Hualien area can provide an earthquake warning within 20 seconds (i.e. crustal shear-wave travelling time over a distance of $120 \mathrm{~km}$ ) after the occurrence of the Hualien earthquake, a timely warning can be feasible for the highly populated Taipei area. Since then Taiwan has been developing EEW system and become one of leading countries on EEW practices. 
The backbone of the present Taiwan EEW system is the Rapid Earthquake Information Release System (RTD; [1, 2]) operated by the Central Weather Bureau (CWB) since 1995. The RTD network at present consists of 109 telemetered seismic stations covering the entire Taiwan region. Each station is equipped with a three-component, force-balance strong-motion accelerometer with a 16-bit resolution and $\mathrm{a} \pm 2 \mathrm{~g}$ full dynamic range recorder.

Although the station density of the RTD network is proven to be rather adequate for repaid reporting and EEW purposes [3], a higher density of seismic stations is always in great demand $[4,5,6]$ especially for a front-detection type of EEW system, in which the seismograms recorded by closer-to-earthquake seismic sensors are used to estimate the earthquake location, magnitude, and to predict the ground motions at more distant target areas. For example, a short-distance EEW system might be possible with extensive installation of seismometers around seismogenic areas [7]. However, to increase the number of seismic station equipped with traditional mechanical seismometer will significantly boost the expense of EEW seismic network. Besides, for the other seismogenic zones around the world where only a limited number of seismic stations or even no seismic network is available, a cost-effective seismic network dedicated to EEW or rapid reporting is highly favored.

The MEMS accelerometer that has been introduced in seismic applications [8] since 1990s is miniature, cost-saving, and ideal for recording high-frequency, near-field unsaturated ground motions. Consequently, the MEMS accelerometer offers a suitable application for an economical, near-source EEW system. The MEMS accelerometer type of network has been proposed for its potential for EEW using the Quake-Catcher Network [9].

The EEW research group at the NTU and the San Lien Technology Corp. (http://www.sanlien.com.tw) have been developing a MEMS type of accelerometer named the "Palert" sensor (Fig. 2.1), specifically designed for EEW purpose. $P$ alert can record three-component acceleration and also can perform real-time integration to obtain velocity and displacement. Once an earthquake $P$ wave is detected by the triggering algorithms embedded in the sensor, the sensor will compute the average period $\left(\tau_{c} ;[10-14]\right)$ and peak amplitude of the filtered vertical displacement (Pd; [12-15]) from the first few seconds of the $P$-wave and accordingly send an earthquake alarm signal for on-site EEW purpose. The triggering algorithms include continuous monitoring of acceleration, displacement, and ShortTerm-Average (STA)/ Long-Term-Average (LTA) ratio [16]. Palert features with networking capability including streaming real-time data to host, automatically connect to up to 2 servers, and Network Time Protocol (NTP) time calibration.

In this study, we will present the EEW performances of the testing Palert EEW network located at the Hualien region (Fig. 2.2), a high seismicity region in eastern Taiwan. The results of the Hualien Palert EEW network during its initial phase of operation strongly encourage the further development for the MEMS accelerometer-type of EEW system. 


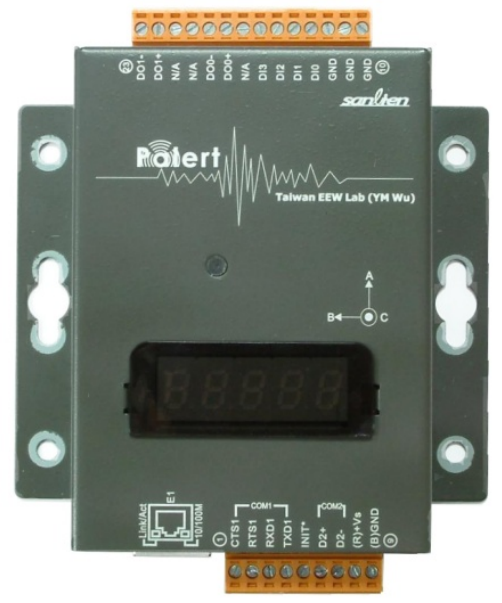

Fig. 2.1 The Palert earthquake early warning sensor.

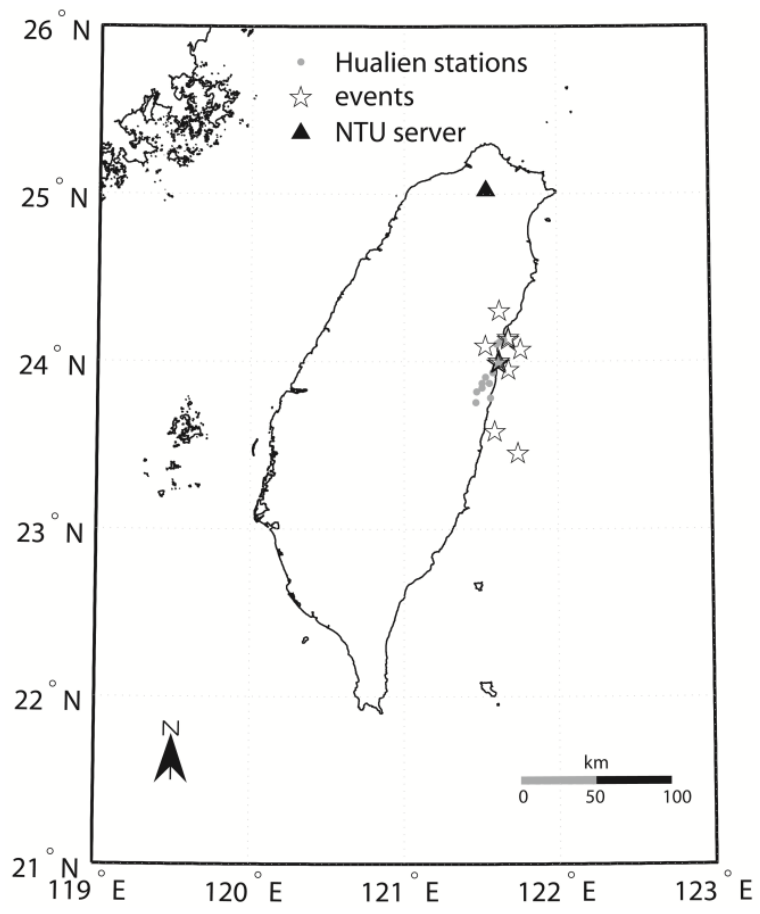

Fig. 2.2 The location map shows the stations (circles) of the Hualien EEW network and the epicenters of the 11 study events (star). The NTU server of the Hualien EEW network is indicated by the triangle. 


\section{The Hualien EEW system}

The Hualien Palert testing EEW network consists of 15 Palert stations, and Fig. 2.3 shows the schematic diagram for the field implementation. There are two main structures involved in the network, consisting of local field systems and a central system. The local system includes signal acquisition and processing by Palert and offers onsite early warning when Pd is larger than $0.35 \mathrm{~cm}$ [7]. The central system includes data clustering and analysis by the network server hosted in Taipei, NTU. The information recorded by the Palert stations is transmitted to the NTU server ocer the TCP/IP protocol. In case of a power failure due to electrical power tower collapses or disconnected communication lines by strong ground shaking, each station is equipped with an internal battery to provide steady electrical power for at least four hours. Once more than 6 Palert stations are triggered, an event is declared. The acquired information from the Palert stations are then stored in the system-wide shared memory and controlled by the code TcPd.c to generate the EEW/rapid reports with event location and magnitude operated by the NTU server.

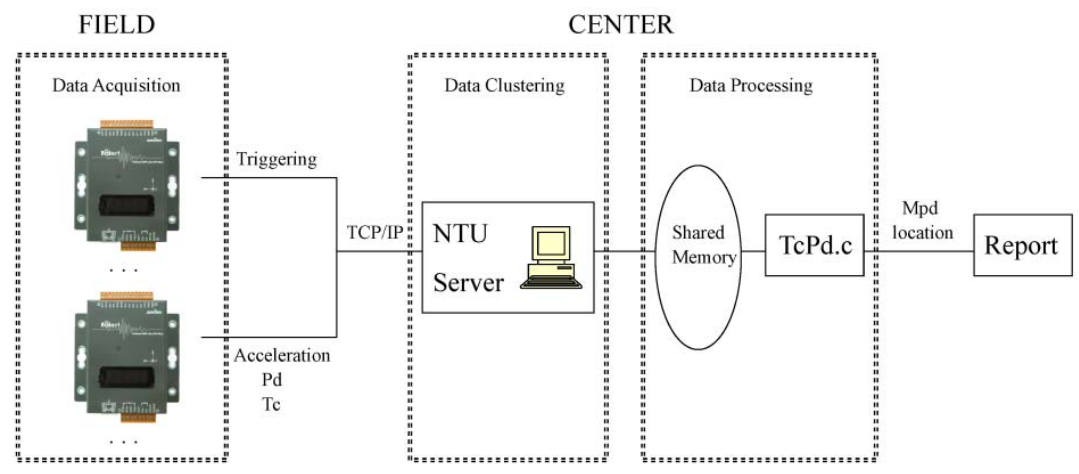

Fig. 2.3 Operation flowchart of the Hualien EEW network.

The hypocentral location will be determined using the traditional earthquake locating algorithm on a half space linear increasing velocity model. The magnitude determination using the Pd attenuation relationship with hypocentral distance $(R)\left(M_{\mathrm{Pd}} ;[17]\right)$, which can be expressed as:

$$
M_{P d}=4.748+1.371 \times \log _{10}(P d)+1.883 \times \log _{10}(R)
$$

is adopted. As more stations are triggered, the event location and magnitude are continuously updated. 


\section{The performance of the Hualien EEWS}

In this testing, 11 earthquakes in the recording period between June, 2010 and August, 2010 and located near the Hualien EEWS are used (Table 1). The choices of the events near the Hualien EEW network follow the proposition as using the Hualien network to be the front-detection sensor. Comparisons of the earthquake information (locations and magnitudes) given by the Hualien EEW system and the CWB published earthquake catalogs are shown in Fig. 2.4. Since a simplified velocity model is used in the code TcPd.c to find the hypocenters and most of the events are located outside the network. So, a large variation of the earthquake locations is indicated in Fig. 2.4. However, for the events with shallow focal depths or located inside the perimeter of the stations the discrepancies of the hypocenters of those between the Hualien EEW system and the CWB catalogs are smaller. The response time as the time difference between the event original time and the first report given by the Hualien EEW system varies between 5 and 25 seconds with the average time of 9.5 second. Therefore, the Hualien EEW system is capable to issue a timely warning to areas located more than $35 \mathrm{~km}$ away from the hypocenter before the arrival of the $S$-wave. The large uncertainty is found in magnitude determination. $M_{\mathrm{Pd}}$ estimated with Equation (1) has a 1:1 relationship with $M_{\mathrm{L}}$ with standard deviations of 1.25 and 1.24 for the first and final reports, respectively. However, earthquakes with magnitudes large than 4.0 have a much better correlation between $\mathrm{P} d$ and the final magnitude.
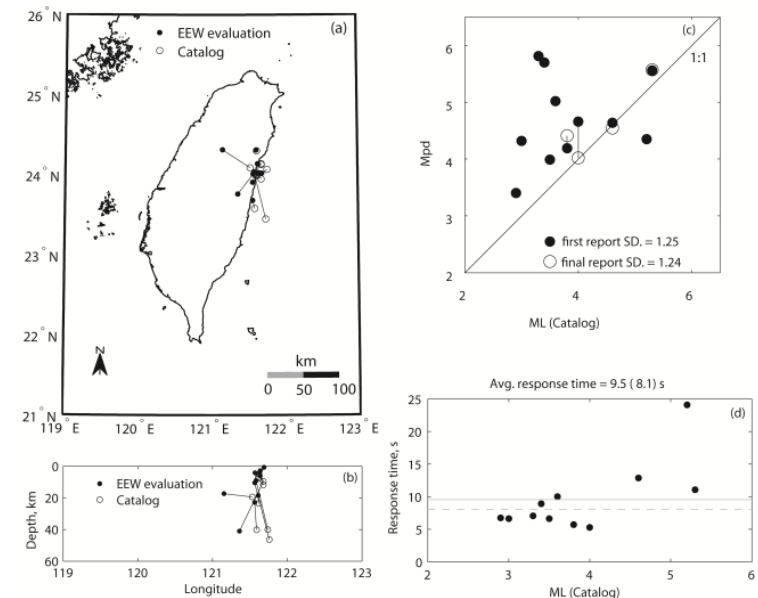

Fig. 2.4 Comparisons of the earthquake information ((a) epicenters, (b) focal depths, (c) magnitudes, (d) response times) given by the real-time, on-line Hualien EEW system and the CWB published earthquake catalogs. In (c), the solid and open circles represent the first report with more than 6 triggered stations and the final report with all available stations, respectively. The response times in (d) are those given by the first report. The average response time of $8.1 \mathrm{~s}$ indicated by the dashed line in (d) is the one excluding the maximum response time of $24.1 \mathrm{~s}$. 


\begin{tabular}{cccccc}
\hline $\begin{array}{c}\text { Event } \\
\text { no. }\end{array}$ & mm/dd/yy & $\begin{array}{c}\text { Origin } \\
\text { time }\end{array}$ & $\begin{array}{c}\text { CWB catalog } \\
\text { Location (lon, lat) }\end{array}$ & Depth $(\mathrm{km})$ & Magnitude $\left(M_{\mathrm{L}}\right)$ \\
1 & $6 / 18 / 2010$ & $04: 24: 06.1$ & $121.68,24.13$ & 9.6 & 2.9 \\
2 & $7 / 04 / 2010$ & $02: 43: 05.0$ & $121.68,23.95$ & 11.8 & 4.0 \\
3 & $7 / 17 / 2010$ & $09: 04: 17.2$ & $121.74,23.45$ & 40.0 & 5.2 \\
4 & $7 / 24 / 2010$ & $23: 41: 10.1$ & $121.62,24.30$ & 23.5 & 3.6 \\
5 & $7 / 28 / 2010$ & $22: 18: 41.2$ & $121.68,24.14$ & 9.7 & 3.3 \\
6 & $7 / 31 / 2010$ & $08: 13: 17.8$ & $121.76,24.07$ & 46.4 & 4.6 \\
7 & $8 / 10 / 2010$ & $21: 57: 51.4$ & $121.53,24.09$ & 19.4 & 3.4 \\
8 & $8 / 19 / 2010$ & $07: 11: 21.3$ & $121.62,24.00$ & 5.1 & 3.5 \\
9 & $8 / 19 / 2010$ & $07: 45: 30.1$ & $121.61,23.99$ & 7.0 & 3.8 \\
10 & $8 / 20 / 2010$ & $06: 46: 23.1$ & $121.62,23.99$ & 7.7 & 3.0 \\
11 & $8 / 21 / 2010$ & $16: 38: 00.0$ & $121.59,23.58$ & 40.2 & 5.3 \\
\hline
\end{tabular}

Table 1a: Source parameters of the eleven earthquakes determined by the Central Weather $\mathrm{Bu}$ reau $(\mathrm{CWB})$

\begin{tabular}{cccccc}
\hline $\begin{array}{c}\text { Event } \\
\text { no. }\end{array}$ & mm/dd/yy & Time & $\begin{array}{c}\text { EEW evaluation } \\
\text { Location } \\
\text { (lon, lat) }\end{array}$ & Depth $(\mathrm{km})$ & Magnitude $\left(M_{\mathrm{pd}}\right)$ \\
1 & $6 / 18 / 2010$ & $04: 24: 12.8$ & $121.63,24.14$ & 4.2 & 3.4 \\
2 & $7 / 04 / 2010$ & $02: 43: 10.3$ & $121.72,23.95$ & 24.9 & 4.7 \\
2 & $7 / 04 / 2010$ & $02: 43: 14.3$ & $121.64,24.02$ & 6.0 & 4.0 \\
3 & $7 / 17 / 2010$ & $09: 04: 41.3$ & $121.58,24.04$ & 9.1 & 4.4 \\
4 & $7 / 24 / 2010$ & $23: 41: 20.1$ & $121.61,24.31$ & 18.5 & 5.0 \\
5 & $7 / 28 / 2010$ & $22: 18: 48.2$ & $121.36,23.76$ & 41.1 & 5.8 \\
6 & $7 / 31 / 2010$ & $08: 13: 30.7$ & $121.57,23.93$ & 12.6 & 4.6 \\
6 & $7 / 31 / 2010$ & $08: 13: 31.7$ & $121.56,23.91$ & 10.6 & 4.6 \\
7 & $8 / 10 / 2010$ & $21: 58: 00.3$ & $121.15,24.31$ & 17.4 & 5.7 \\
8 & $8 / 19 / 2010$ & $07: 11: 27.9$ & $121.64,24.03$ & 2.9 & 4.0 \\
9 & $8 / 19 / 2010$ & $07: 45: 35.8$ & $121.57,24.01$ & 4.2 & 4.2 \\
9 & $8 / 19 / 2010$ & $07: 45: 37.8$ & $121.58,24.01$ & 3.3 & 4.4 \\
9 & $8 / 19 / 2010$ & $07: 45: 40.8$ & $121.57,24.01$ & 4.2 & 4.4 \\
10 & $8 / 20 / 2010$ & $06: 46: 29.7$ & $121.69,24.02$ & 0.7 & 4.3 \\
11 & $8 / 21 / 2010$ & $16: 38: 11.1$ & $121.55,23.61$ & 29.9 & 5.7 \\
11 & $8 / 21 / 2010$ & $16: 38: 12.1$ & $121.56,23.69$ & 21.6 & 5.6 \\
11 & $8 / 21 / 2010$ & $16: 38: 13.1$ & $121.56,23.68$ & 22.9 & 5.6 \\
\hline
\end{tabular}

* Multiple event numbers indicate that more than one report is made for that event.

Table 1b: Source parameters of the eleven earthquakes determined by the Hualien EEW system.

\section{Discussion and conclusions}

In addition to the physical properties of the MEMS accelerometer, the main advantage of the MEMS accelerometer comparing to the other seismometers is that it is a relatively very low-cost seismic sensor. For the commercial type of Palert 
the cost is generally less than thousand US dollar. A seismic network comprising couple tens or even hundreds of the Palert sensors can be built by a much lower budget than that with the transitional seismometers.

The differences of the hypocentral locations between the reports by the Hualien EEWS and the CWB catalogs are partly attributed to the simplified velocity model used in the Hualien EEW location algorithm and to the linear station distribution in the N-S direction of the Hualien EEW network. Another source of deviation might be caused by the inaccurate automated first-arrive picking especially for the smaller earthquakes. In the future development of the Hualien EEW system, a 1-D velocity model with more layers might be adopted. Eventually, for the final phase of EEW network development, a low-cost and densely distributed Palert-type network over the whole Taiwan region is feasible. Such extensively and densely distributed network is especially useful for a large inland earthquake or with a long rupture length, which is often more capable of causing severe damage than outland ones in Taiwan.

The discrepancies between the predicted $M_{\mathrm{Pd}}$ and the catalog $M_{\mathrm{L}}$ might arise from poor earthquake location and consequently incorrect distance term in implementing Pd attenuation relationship (Fig. 2.4c). Fig. 2.5a shows that the standard deviation (1.24) shown in Fig. 2.4c is reduced to 1.06 by replacing the EEWdetermined location with the catalog location. The region-depend Pd attenuation relationship [17] used in this study (Equation 1) is derived for earthquakes in Southern California and there was no such relationship available during the construction phase of the Hualien EEW network. Fig. 2.5b compares $M_{\mathrm{Pd}}$ calculated by the newly derived Taiwan Pd attenuation relationship [18] with the catalog $M_{\mathrm{L}}$. The standard deviation (1.24) shown in Fig. 2.4c is significantly reduced to 0.64 without an overestimated bias as shown in Figs. $2.4 \mathrm{c}$ and 2.5a. A local Pd attenuation relationship specifically for the Hualien EEW network might further improve the EEW performance in magnitude determination. Another noticeable feature of the Hualien EEW network is the response time. For the current operating EEW system by CWB the average response time is of about 20 second [18].
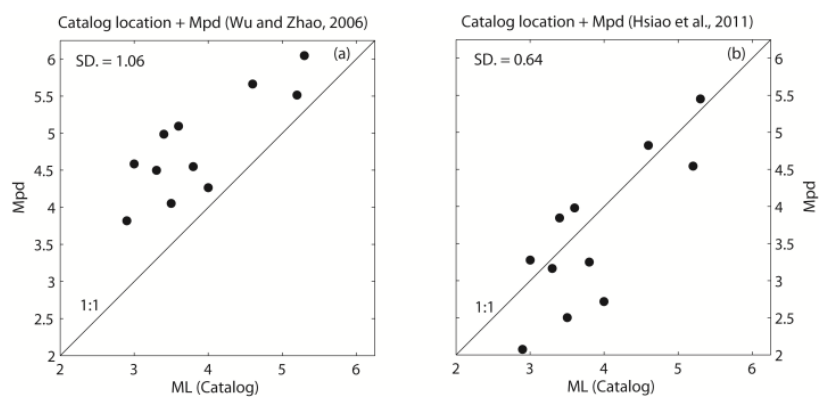

Fig. 2.5 Magnitude $\left(M_{\mathrm{P} d}\right)$ determined by the Hualien EEW network by using (a) the southern California (Wu and Zhao [17]) and (b) the Taiwan Pd attenuation relationships (Hsiao et al. [18]), respectively, versus $M_{\mathrm{L}}$ in the CWB earthquake catalogs. Both in (a) and (b) the earthquake locations are given by the CWB earthquake catalogs. 
For magnitude determination problem in EEW system, a large event with large rupture dimensions, such as the $M_{\mathrm{w}}=9.02011$ Tohoku earthquake, likely consists of several strong asperities over the entire rupture volume. Using the first few seconds of the waveform from an initial nucleation rupture for estimating the eventual magnitude, which is the concept behind the EEW system, may be difficult for extremely large earthquakes. However, recently result [19] indicates that the effective shaking method of $\mathrm{Wu}$ and Teng [20] provides excellent magnitude estimation for the 2011 Tohoku earthquake, without a saturation problem. Such approach could be used in Palert network for updating magnitude determination. Thus, within few to ten seconds and few minutes Pd and effective shaking methods could be used for EEW and rapid reporting purposes, respectively.

Our new EEW sensor, Palert, using the MEMS sensor has a high commercial potential owing to its low cost. The EEW system could be established in a relatively low budge or Palert sensor could be readily added to the existing seismic network to increase the density of the network. The Hualien EEW system belongs to the front-detection type. Nevertheless, our Palert sensor also can be used in the on-site type of EEWS, which the initial $P$-wave motion at a target site is used to predict the ground motions of the later $S$ and surface waves at the same site.

Acknowledments:

Our work was supported by the National Science Council and Central Weather Bureau, Taiwan. We thank Prof. Friedemann Wenzel, Dr. Hanshu Peng and two anonymous reviewers for constructing comments.

\section{References}

[1] Wu YM, Chen CC, Shin TC, Tsai YB, Lee WHK, Teng TL (1997) Taiwan Rapid Earthquake Information Release System. Seism Res Lett 68:931-943

[2] Wu YM, Lee WHK, Chen CC, Shin TC, Teng TL, Tsai YB (2000) Perfor-mance of the Taiwan Rapid Earthquake Information Release System (RTD) during the 1999 Chi-Chi (Taiwan) earthquake. Seismo Res Let 71:338-343

[3] Hsiao NC, Wu YM, Shin TC, Zhao L, Teng TL (2009) Development of earthquake early warning system in Taiwan. Geophys Res Lett. doi:10.1029/2008GL036596

[4] Lin TL, Wu YM (2010) Magnitude determination using strong ground-motion attenuation in earthquake early warning. Geophys Res Lett. doi:10.1029/2010GL042502

[5] Lin TL, Wu YM (2010) Magnitude estimation using the covered areas of strong ground motion in earthquake early warning. Geophys Res Lett. doi:10.1029/2010GL042797

[6] Lin TL, Wu YM, Chen DY (2011) Magnitude estimation using initial P-wave amplitude and its spatial distribution in earthquake early waning in Taiwan. Geophys Res Lett. doi:10.1029/2011GL047461

[7] Wu YM, Lin TL, Chao WA, Huang HH, Hsiao NC, Chang CH (2011) Faster short-distance earthquake early warning using continued monitoring of filtered vertical displacement - a case study for the 2010 Jiasian earthquake, Taiwan. Bull Seism Soc Am. doi: $10.1785 / 0120100153$

[8] Holland A (2003) Earthquake Data Recorded by the MEMS Accelerometer. Seismol Res Lett $74: 20-26$

[9] Cochran ES, Lawrence JF, Christensen C, Jakka RS (2009) The Quake-Catcher Network: Citizen science expanding seismic horizons. Seismol Res Lett 80:26-30. 
[10] Kanamori H (2005) Real-time seismology and earthquake damage mitigation. Annual Review of Earth and Planetary Sciences. doi:10.1146/annurev.earth.33.092203.122626

[11] Wu YM, Kanamori H (2005) Experiment on an onsite early warning method for the Taiwan early warning system. Bull Seismol Soc Am 95:347-353

[12] Wu YM, Kanamori H, Allen R, Hauksson E (2007) Determination of earthquake early warning parameters, $\tau \mathrm{c}$ and Pd, for southern California. Geophys J Int. doi:10.1111/j.1365246X.2007.03430.x

[13] Wu YM, Kanamori H (2008) Development of an Earthquake Early Warning System Using Real-Time Strong Motion Signals. Sensors 8:1-9

[14] Wu YM, Kanamori H (2008) Exploring the feasibility of on-site earthquake early warning using close-in records of the 2007 Noto Hanto earthquake. Earth Planets and Space 60:155160

[15] Wu YM, Kanamori H (2005) Rapid assessment of damaging potential of earthquakes in Taiwan from the beginning of P waves. Bull Seismol Soc Am 95:1181-1185

[16] Allen RV (1978) Automatic earthquake recognition and timing from single traces. Bull Seismol Soc Am 68:1521-1532

[17] Wu YM, and L. Zhao (2006) Magnitude estimation using the first three seconds P-wave amplitude in earthquake early warning. Geophys Res Lett. doi:10.1029/2006GL026871

[18] Hsiao NC, Wu YM, Zhao L, Chen DY, Huang WT, Kuo KH, Shin TC, Leu PL (2011) A new prototype system for earthquake early warning in Taiwan. Soil Dyn Earthquake Eng. doi:10.1016/j.solidyn.2010.01.008

[19] Lin TL, Wu YM (2012) A fast magnitude estimation for the M 9.02011 Great Tohoku Earthquake. Sismo. Res. Let. in press

[20] Wu YM, TL Teng (2004) Near Real-Time Magnitude Determination for Large Crustal Earthquakes. Tectonophysics 390:205-216 